\title{
Miscibility of Polysulfone/Poly(1-vinylpyrrolidone-co-styrene) Blends and Their Application to the Ultrafiltration Membrane
}

\author{
Joo Heun Kim, Jung Eun Yoo, and Chang Keun Kim* \\ Department of Chemical Engineering, Chung-Ang University, 221 Huksuk-Dong, Dongjak-Gu, Seoul 156-756, Korea
}

Received May 21, 2002; Revised July 10, 2002

\begin{abstract}
Miscibility of polysulfone (PSf) with various hydrophilic copolymers was explored. Among these blends, PSf gives homogeneous mixtures with poly(1-vinylpyrrolidone-co-styrene) copolymers [P(VP-S)] when these copolymers contained VP from 68 to $88 \mathrm{wt} \%$. Microporous membranes for the ultrafiltration process were prepared from PSf blends with P(VP-S) copolymers. The membranes prepared from the PSf/P(VP-S) blends exhibited higher water flux than the membranes prepared from PSf irrespective of the VP content. The solute rejection examined with the membranes fabricated from the miscible blends was similar to that of PSf membrane. However, the solute rejection examined with the membranes fabricated from the immiscible blends was lower than that of PSf membranes.

Keywords : polysulfone, poly(1-vinylpyrrolidone-co-styrene) copolymer, blend, miscibility, ultrafiltration, membrane performance.
\end{abstract}

\section{Introduction}

Polysulfones are using in a variety of applications because of its excellent balance of properties. ${ }^{1}$ Polysulfones are clear, rigid, and tough thermoplastics with the high glass transition and the chemical inertness. The high thermal resistance combined with excellent hydrolytic stability and the ability to retain mechanical properties in hot, wet environments are the keys to their use which require repeated cleaning with hot water or sterilization. Bisphenol-A polysulfone (PSf) is used in a membrane support for reverse osmosis, ultrafiltration, and gas separation. However, the hydrophobic properties of polysulfones deter their application in the membrane area. The hydrophobic surface of PSf membranes has a severe fouling during the ultrafiltration process caused by hydrophobic interactions between solute and PSf. A number of approaches have been used to change the hydrophobic PSf membrane to the hydrophilic membrane such as surface treatment with plasma. ${ }^{2-6}$ Poly (vinylpyrrolidone) (PVP) often mixed with the PSf casting solution to provide hydrophilic properties to the PSf membrane. However, this approach was not effective because PVP is washed out to the water phase during the phase inversion process that has been widely used for the fabrication of microporous membrane. ${ }^{7}$

Blends of PSf with hydrophilic polymer that is not water

*e-mail : ckkim@cau.ac.kr

1598-5032/08/209-06 (C) 2002 Polymer Society of Korea soluble might provide products having improved hydrophiliciity in comparison to PSf. Polymer blend exhibited separated phase structure when its interaction energy is positive at the processing temperature and pressure. The application of immiscible blends often limited because of their poor adhesion strength at interface. Unlike other miscible polymer blends, miscible PSf blends do not reported as far as we know to date. It is known that PSf does not form miscible blends with common polymers such as polystyrene (PS) and polymethylmethacrylate (PMMA) and hydrophilic polymers such as PVP and polyvinylalcohol (PVA). ${ }^{7-9}$ Small variations in the molecular structure of one component may cause large changes in the phase behavior of blends. According to a simple binary interaction model for describing how the net interaction energy depends on copolymer composition, copolymerization can be an effective strategy to produce a new miscible blend. ${ }^{10-13}$

In current work, we examined PSf blends with various hydrophilic copolymers to produce miscible blends that can be used for membrane materials having hydrophilic properties. The performance of ultrafiltration membranes fabricated from the blends of PSf with the hydrophilic copolymers was explored.

\section{Materials and Procedures}

Polymers used in this study were listed in Table I. Commercially available PSf, Udel, P-3500, from Amoco Performance Products Inc., was used. In this wok, copolymers such 
Table I. Polymers Used in This Study

\begin{tabular}{lcccl}
\hline Abbreviation $^{a}$ & $\begin{array}{c}\text { VP Contents } \\
(\text { wt } \%)^{b}\end{array}$ & $T_{g}\left({ }^{\circ} \mathrm{C}\right)$ & $\bar{M}_{w}{ }^{c}$ & Source \\
\hline PSf & - & 189 & 69,000 & $\begin{array}{l}\text { Amoco } \\
\text { performance }\end{array}$ \\
PEG & - & - & 20,000 & Polyscience \\
P(VP-S) 25 & 23.5 & 107 & 65,000 & Synthesized \\
P(VP-S) 32 & 32.4 & 109 & 42,000 & Aldrich \\
P(VP-S) 35 & 36.1 & 115 & 70,500 & Synthesized \\
P(VP-S) 50 & 48.7 & 123 & 62,000 & Synthesized \\
P(VP-S) 60 & 58.9 & 130 & 71,000 & Synthesized \\
P(VP-S) 65 & 65.1 & - & 59,000 & Synthesized \\
P(VP-S) 68 & 68.6 & 134 & 79,000 & Synthesized \\
P(VP-S) 70 & 72.4 & 137 & 75,000 & Synthesized \\
P(VP-S) 75 & 77.3 & 140 & 80,500 & Synthesized \\
P(VP-S) 80 & 81.1 & 148 & 77,000 & Synthesized \\
P(VP-S) 88 & 88.4 & - & 69,000 & Synthesized \\
P(VP-S) 90 & 92.1 & 153 & 61,000 & Synthesized \\
P(VP-S) 95 & 96.3 & 155 & 67,000 & Synthesized \\
\hline
\end{tabular}

${ }^{a}$ The numerical value included as part of the code for copolymers indicates the weight percent of VP.

${ }^{b}$ The VP content in the copolymer was determined by element analysis. ${ }^{c}$ Molecular weight were determined by GPC with polystyrene standards.

as poly(1-vinylpyrrolidone-co-styrene) [P(VP-S)], poly(vinylacetate-co-styrene) [P(VAc-S)], poly(vinylalcohol-co-styrene) [P(VA-S)], and poly(vinylalcohol-co-methyl methacrylate) [P(VA-MMA)] were synthesized at various copolymer compositions. Among these coplymers, P(VP-S) copolymers containing certain amounts of styrene only formed miscible blend with polysulfones. Because of this, the synthetic procedure for the P(VP-S) copolymer was only described here. The synthesis of $\mathrm{P}(\mathrm{VP}-\mathrm{S})$ copolymers was performed in the bulk at $80^{\circ} \mathrm{C}$ with AIBN (azobisisobutylronitrile) as the initiator and ethyl benzene as the chain transfer agent. Polymerization was stopped at an early stage, usually below 15 wt $\%$ conversion, to minimize composition drift. The resulting solution was poured into a large excess of n-hexane to precipitate the polymer. The precipitated polymer was vacuum filtered, air dried overnight and dried in a vacuum oven at $80^{\circ} \mathrm{C}$ for $24 \mathrm{hrs}$. Molecular weight information of polymers was obtained using GPC calibrated with polystyrene stand ards, and monomer content of copolymers was determined by elemental analysis. The numerical value included as part of the code for these copolymers indicates the weight percent of VP.

Two different methods coded $\mathrm{C}_{1}$ and $\mathrm{C}_{2}$ were used to prepare PSf blends with copolymer. The details of each are described below. $\mathrm{C}_{1}$ : In this method, PSf and copolymer were precipitated simultaneously from the 1-methyl-2-pyrrolidone (NMP) solution using the nonsolvent water. Note that this method is similar to the wet phase inversion process for the fabrication of the ultrafiltration membrane. The precipitate was allowed to dry in an air-circulating oven and then further dried in a vacuum oven at $140^{\circ} \mathrm{C}$ for a week. $\mathrm{C}_{2}$ : The blend was prepared in film form by casting solutions containing $5 \mathrm{wt} \%$ total polymer in NMP onto petri dish at $130^{\circ} \mathrm{C}$. After slow drying at room temperature, the blends were finally dried in a vacuum oven at $140^{\circ} \mathrm{C}$ for a week.

Thermal analysis was performed at a heating rate of $20^{\circ} \mathrm{C} / \mathrm{min}$ by using a DSC (TA Instrument, DSC-2010). The first scan was run to $220^{\circ} \mathrm{C}$ to erase previous thermal history during sample preparation and storage, then the sample was quenched to $25^{\circ} \mathrm{C}$ to start the second scan. The glass transition temperature, $T_{g}$, was defined as the onset of the change in heat capacity during the second heating from 25 to $250^{\circ} \mathrm{C}$.

Membranes for the ultrafiltration were prepared by the wet phase inversion process that is a well-known process for the preparing a variety of asymmetric membranes., ${ }^{74-16}$ Dimethylformamide (DMF), dimethylsulfoxide (DMSO), and NMP are generally used as solvents for the preparation of casting solution of PSf. Since P(VP-S) copolymers were not dissolved in the DMF and DMSO, NMP was used as solvent for the casting solution. Polymer solution containing PSf, P(VP-S), and NMP was cast onto the nonwoven polyester fabrics using a doctor blade with the thickness of $0.15 \mathrm{~mm}$. The cross-sectional morphologies of membranes were examined using scanning electron microscopy (SEM, Hitachi, model S-2500). The samples were fractured in liquid nitrogen and coated with gold by ion sputtering. The casting solution was immediately precipitated by immersion in a water bath. An aqueous solution containing 1,000 ppm of PEG 20000 (polyethyleneglycol, weight average molecular weight $=$ 20,000 , polydisperse index $=1.03$ ) was used as the feed solution for the membrane performance test. The permeate flux was measured at $3 \mathrm{bar}$, flow rate of $2.5 \mathrm{~L} / \mathrm{min}$, and $25^{\circ} \mathrm{C}$. The solute concentration of the permeate was measured by using HPLC equipped with a refractomer.

\section{Results and Discussion}

Miscibility of Blends. The blends of PSf with copolymers such as $\mathrm{P}(\mathrm{VAc}-\mathrm{S}), \mathrm{P}(\mathrm{VA}-\mathrm{S})$, and $\mathrm{P}(\mathrm{VA}-\mathrm{MMA})$ were immiscible regardless of the copolymer compositions and preparation methods. The blends were translucent and their thermograms revealed two $T_{g} \mathrm{~s}$. Because of the immiscibility of these copolymers with PSf, P(VP-S) copolymers were only used for the further study. Blends of PSf with P(VP-S) copolymers having VP contents from $25 \%$ up to $95 \%$ by weight were explored.

The PSf/P(VP-S) blends casted from NMP at $130^{\circ} \mathrm{C}$ were transparent and their thermograms reveal a single $T_{g}$ when 


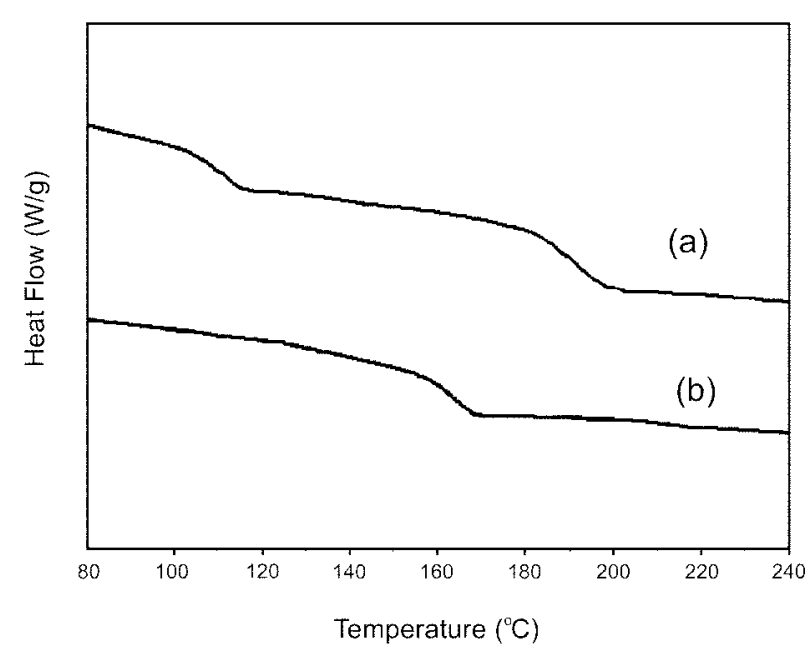

Figure 1. DSC thermograms of PSf blends with $\mathrm{P}(\mathrm{VP}-\mathrm{S})$ copolymers; (a) PSf/P(VP-S) $32=6 / 4$; (b) PSf/P(VP-S) $80=6 / 4$.
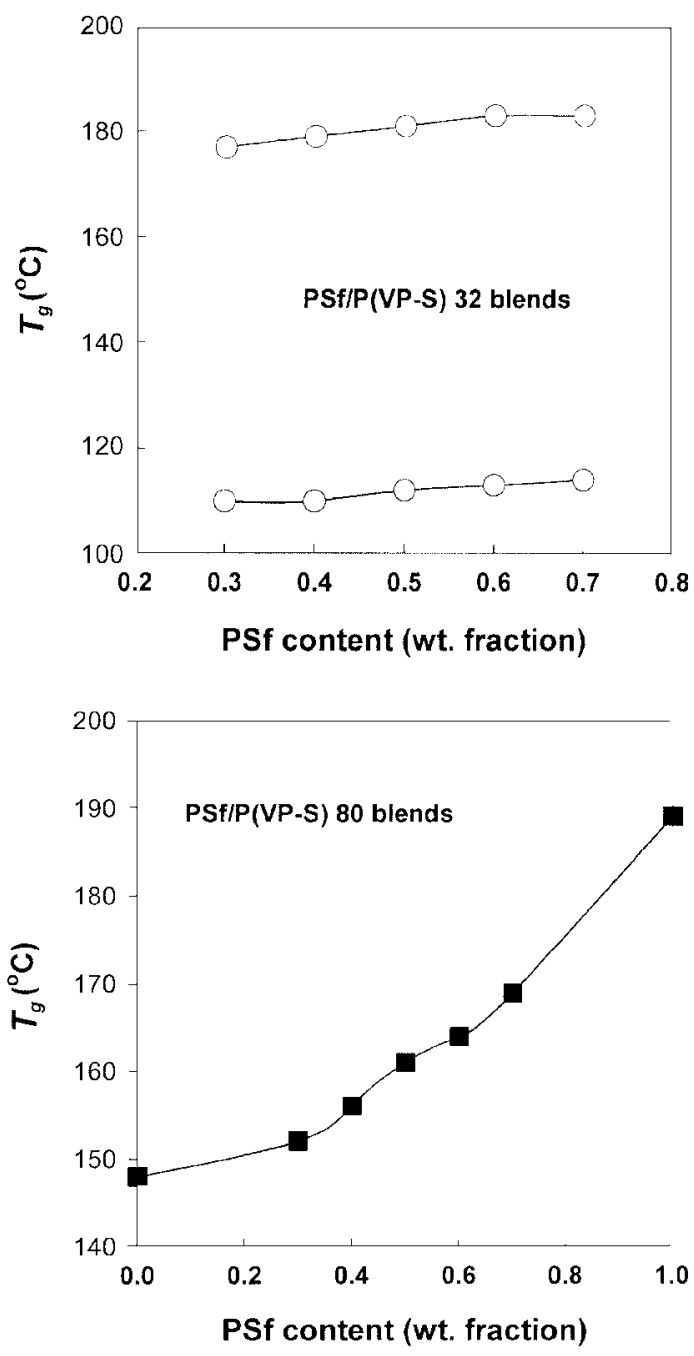

P(VP-S) copolymers contain VP from 68 to $88 \mathrm{wt} \%$. However, PSf blends with the $\mathrm{P}(\mathrm{VP}-\mathrm{S})$ copolymers containing $65 \mathrm{wt} \% \mathrm{VP}$ and those with the P(VP-S) copolymers containing $90 \mathrm{wt} \% \mathrm{VP}$ were opaque and showed two $T_{g} \mathrm{~s}$ indicating that phase separation occurred. Figure 1 exhibits selected DSC thermograms for PSf/P(VP-S) 32 blend and PSf/P(VP-S) 80 blend. Miscible blend of PSf and P(VP-S $)$ 80 showed a single $T_{g}$ while immiscible blend of PSf and P(VP-S) 32 showed two separated $T_{g}$ s. Figure 2 shows representative results of glass transition temperatures for PSf blends P(VP-S) copolymers having VP contents from 32 up to $95 \%$ by weight. Hot cast blends of PSf with copolymers having VP contents from 68 to $88 \mathrm{wt} \%$ showed a single $T_{g}$ that varied regular with overall blend composition. The glass transition behavior for blends containing $50 \mathrm{wt} \%$ of PSf was summarized in Figure 3 by plotting the observed glass transitions versus VP content of the copolymer. PSf/P(VPS) blends were also prepared by dissolving in NMP and then
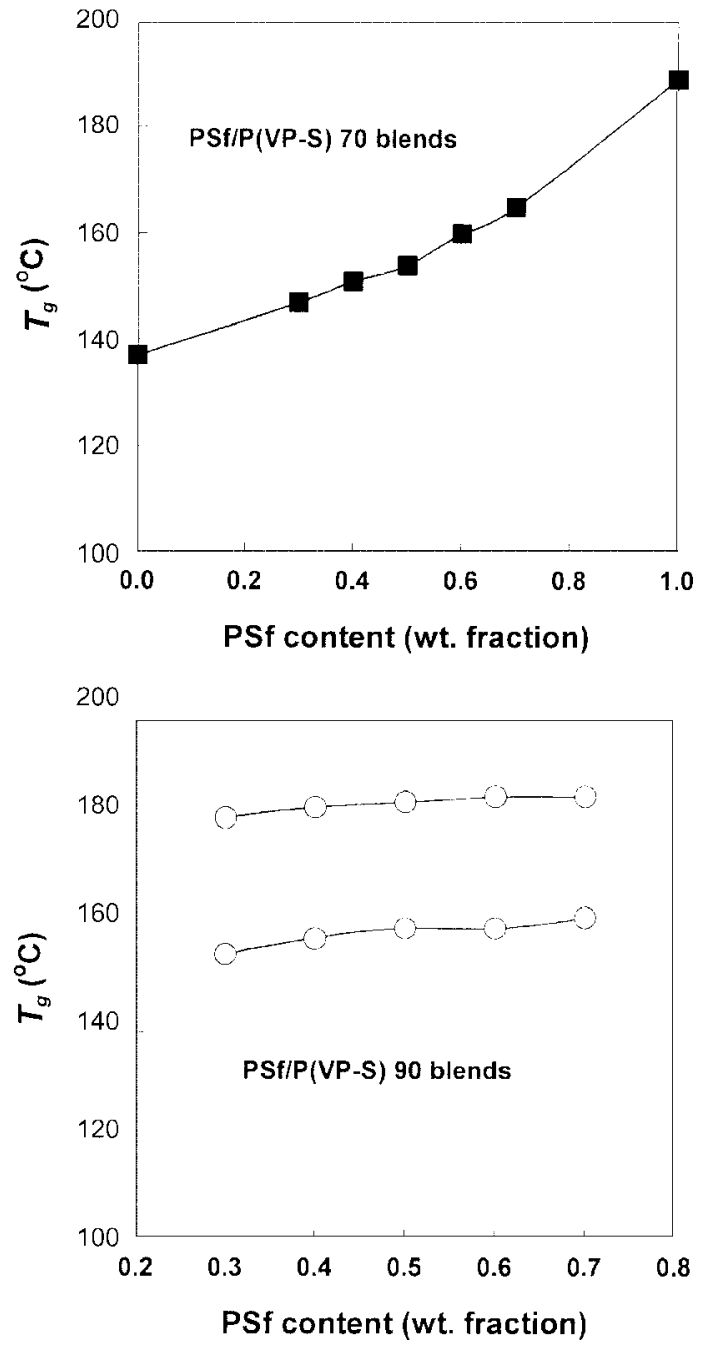

Figure 2. Glass transition behavior for PSf blends with various P(VP-S) copolymers. 


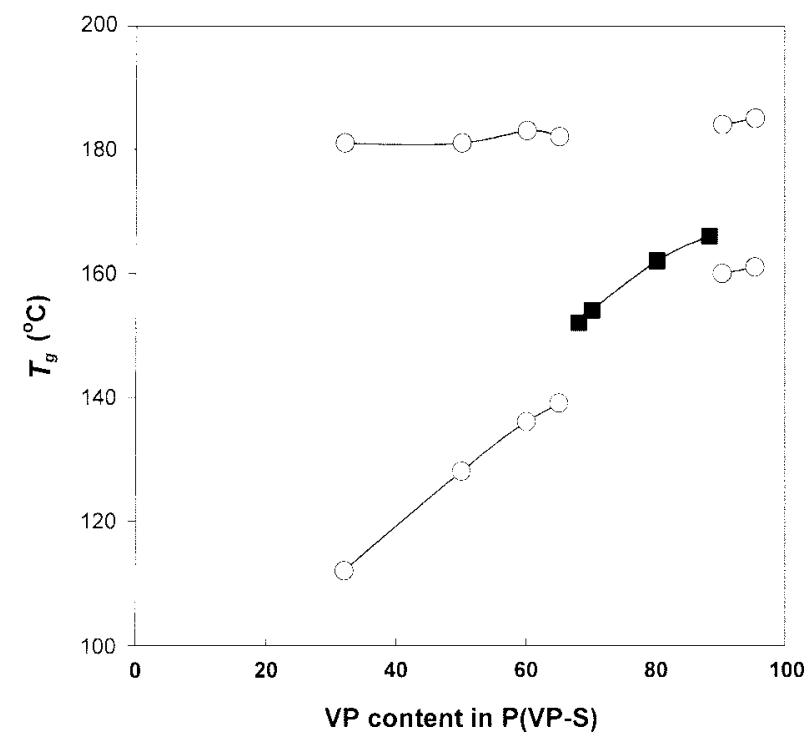

Figure 3. Glass transitions for blends containing $50 \mathrm{wt} \% \mathrm{PSf}$ and $50 \mathrm{wt} \% \mathrm{P}(\mathrm{VP}-\mathrm{S})$ copolymer versus VP content of copolymer.

pouring the solution into water to precipitate them simultaneously (method $\mathrm{C}_{1}$ ). The $T_{g}$ behavior of PSf/P(VP-S) blend prepared by method $\mathrm{C}_{1}$ was similar to that prepared by method $\mathrm{C}_{2}$. From these results, it was concluded that PSf and $\mathrm{P}(\mathrm{VP}-\mathrm{S})$ copolymers formed miscible blends when copolymers have VP from 68 to $88 \mathrm{wt} \%$ and beyond these critical limits to form immiscible blends.

The potential effects of copolymer on blend phase behavior can be easily explained by aid of a simple binary interaction model for describing how the interaction energy between homopolymer-copolymer, B, depends on copolymer composition. $^{10-13}$

$$
B=B_{13} \phi_{1}^{\prime}+B_{23} \phi_{2}^{\prime}-B_{12} \phi_{1}^{\prime} \phi_{2}^{\prime}
$$

Here we assume that 1 and 3 are repeat unit of polysulfone and VP, respectively and 2 is styrene monomer that is to be copolymerized with VP. The $\phi_{i}^{\prime}$ are the volume fractions of these units in the copolymer. Since binary pairs involved here, i.e., PSf-PVP, PSf-PS, and PVP-PS are immiscible, interaction energy of each binary pair is positive. According to the equation (1), $B$ can be negative when $B_{12}$ is relatively large positive. Since polymer blend is miscible when $B$ is negative, miscibility of PSf/P(VP-S) blends at a certain copolymer composition range comes from the intramolecular repulsion between VP and styrene. Interaction energies of binary pairs involved in the miscible blends should be characterized for the quantitative understanding of the observed phase behavior. For this analysis, the data such as phase boundary and pressure-volume-temperature behavior of each polymer are required. Quantitative analysis of interaction energies involved in the blends will be a topic of the coming paper.

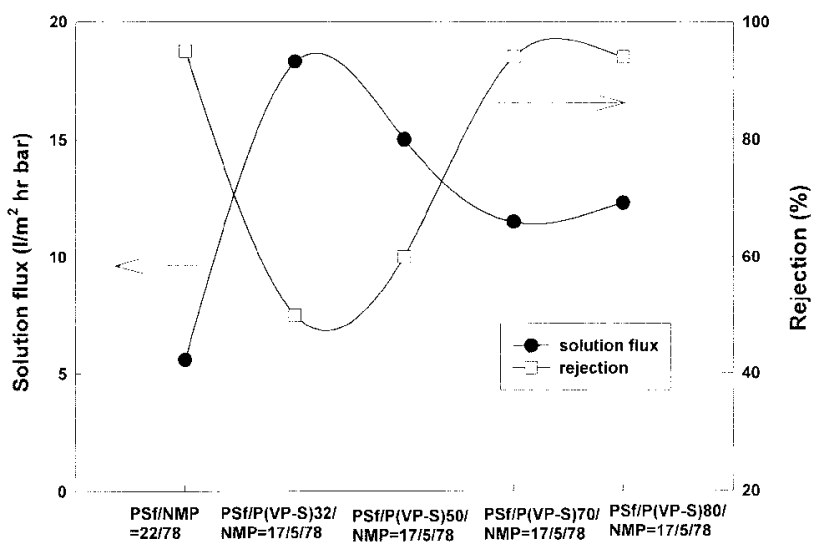

Figure 4. The performance of membranes prepared from PSf and $\mathrm{PSf} / \mathrm{P}(\mathrm{VP}-\mathrm{S})$ blends.

Membrane Performance. Membrane prepared from PSf/NMP (=22/78) solution was used as a standard sample for the performance test. The polymer content of all casting solutions was kept to $22 \mathrm{wt} \%$. The performance of PSf membrane was compared with that of membranes cast from $\mathrm{PSf} / \mathrm{P}(\mathrm{VP}-\mathrm{S}) / \mathrm{NMP}=17 / 5 / 78$ solutions. Figure 4 shows the solution fluxes and the rejection of PEG 20000 examined with five different membranes. The blend membranes might be divided into two catagories, i.e., membranes from the miscible blends and those from the immiscible blends. The PEG rejection of membranes from the miscible blends (PSf/ $\mathrm{P}(\mathrm{VP}-\mathrm{S}) 70$ and PSf/P(VP-S) 80) was similar to that of PSf membrane while the solution flux of membranes from the miscible blends was about two times higher than that of PSf membrane. Membranes from the immiscible blends (PSf/ $\mathrm{P}(\mathrm{VP}-\mathrm{S}) 32$ and PSf/P(VP-S) 50) exhibited higher solution flux than membranes from the miscible blends but their PEG rejection was reduced up to $50 \%$. Since PSf/P(VP-S) 70 contains more hydrophilic component than PSf/P(VP-S) 32, it might be expected that PSf/P(VP-S) 70 membrane give high solution flux. The reduction of PEG rejection examined with PSf/P(VP-S) 32 membrane also appear to be inconsistent with expectation. The cross-sectional morphologies of membranes were observed to rationalize the apparent anomaly.

It is known that the cross-sectional structure of asymmetric polymer membrane depends on the solvent used. ${ }^{7}$ A fingerlike structure was formed when NMP/water was used as the solvent/nonsolvent pair with various polymers. Figure 5 shows the cross-sectional morphologies of membranes prepared from the three different casting solutions. All three membranes that were prepared from the casting soution containing NMP exhibited a finger-like structure as expected. The morphology of PSf/P(VP-S) 70 membrane is similar with that of PSf membrane. Separated phase structure of PSf-rich phase and P(VP-S)-rich phase was not observed because of miscibility of PSf and P(VP-S) 70 copolymer. 


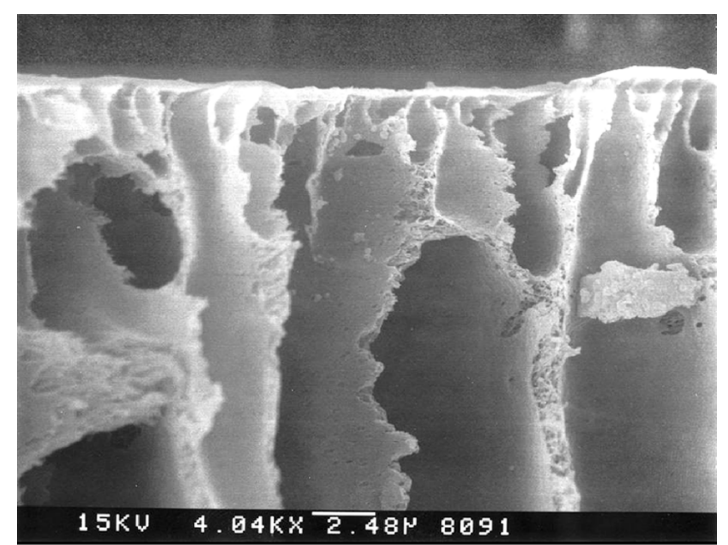

(a)

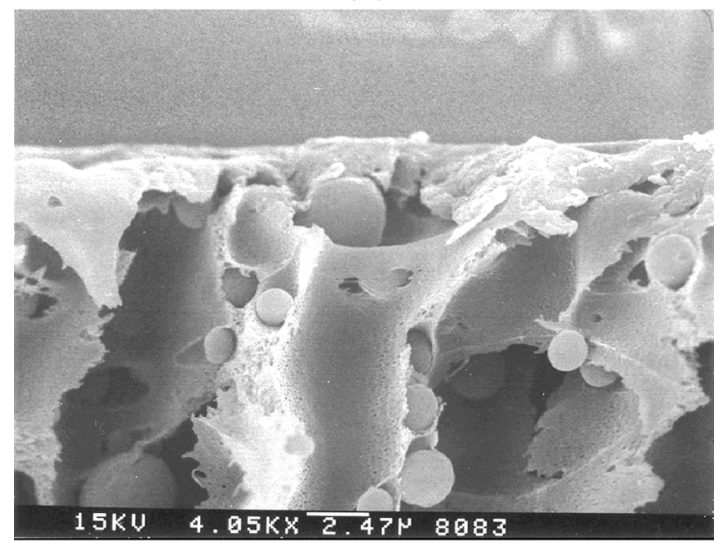

(b)

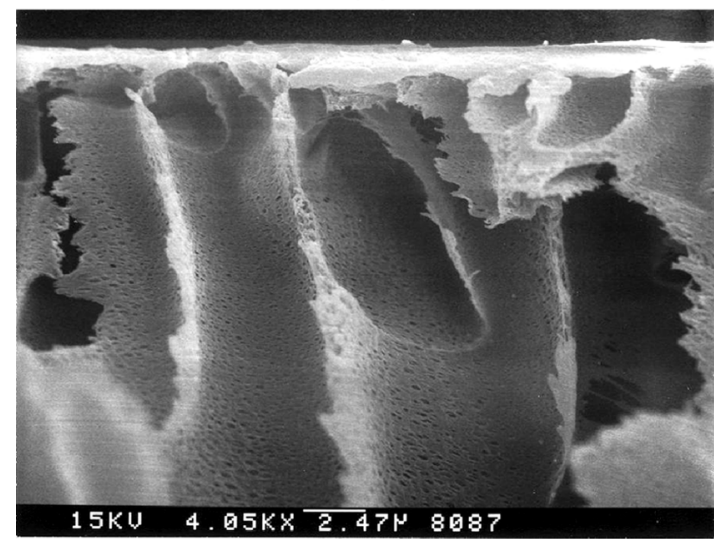

(c)

Figure 5. Cross-sectional morphologies of asymmetric membranes prepared by wet phase inversion process; (a) PSf/NMP = 22/78; (b) PSf/P(VP-S) 32/NMP = 17/5/78; (c) PSf/P(VP-S) 70/ $\mathrm{NMP}=17 / 5 / 78$

High solution flux of PSf/P(VP-S) 70 membrane compared with PSf membrane might stem from hydrophilic property of $\mathrm{P}(\mathrm{VP}-\mathrm{S})$ copolymers. However, membrane prepared from PSf/P(VP-S) 32 blend shows separated phase structure having poor adhesion at polymer-polymer interface formed during precipitation process. The poor adhesion at interface

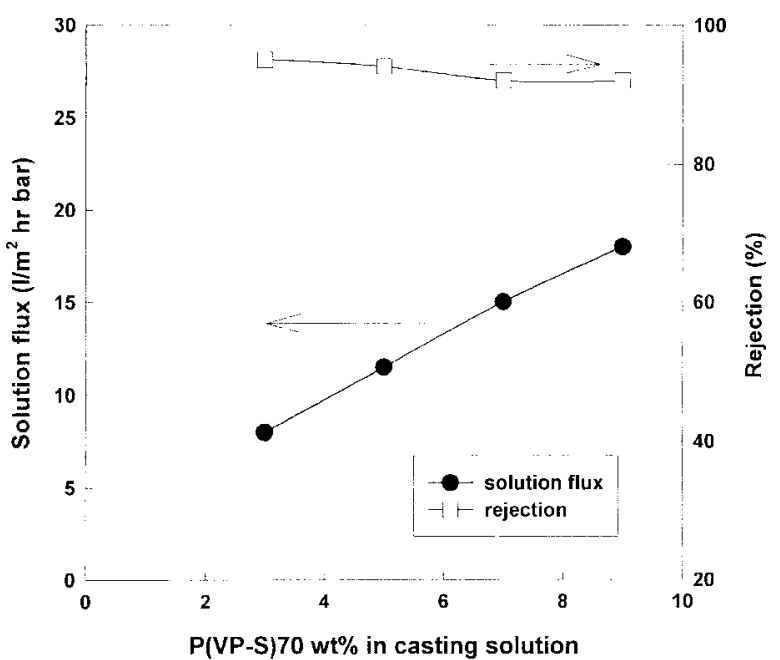

Figure 6. The performance of ultrafiltration membranes prepared from the miscible blends of PSf and P(VP-S) 70 copolymer. Note that all casting solutions contain $22 \mathrm{wt} \%$ polymer.

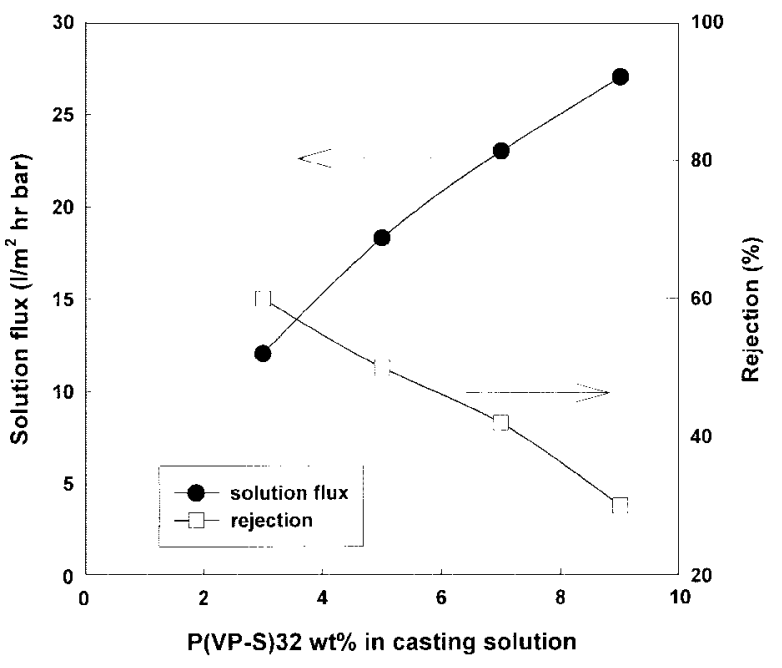

Figure 7. The performance of ultrafiltration membranes prepared from the immiscible blends of PSf and P(VP-S) 32 copolymer. Note that all casting solutions contain $22 \mathrm{wt} \%$ polymer.

might result in decline in PEG rejection and increase in the water flux.

The performace of membranes prepared from the miscible blends of PSf and P(VP-S) 70 was examined by changing blend composition of the casting solution. As shown in Figure 6 , the solute rejection of blend membranes was similar to that of PSf membrane while the solution flux increased in comparision to P(VP-S) 70 content of the casting solution. However, the solute rejection of the membranes from the immiscible blends of PSf and P(VP-S) 32 continuously decreased from 95 to $30 \%$ by increasing $\mathrm{P}(\mathrm{VP}-\mathrm{S})$ content, as shown in Figure 7. These results suggested that the mem- 
branes fabricated from the miscible blends of PSf and $\mathrm{P}(\mathrm{VP}-\mathrm{S})$ showed the performance advantage over PSf membrane.

\section{Summary}

Miscibility of PSF with various hydrophilic copolymers such as P(VP-S), P(VAc-S), P(VA-S), and P(VA-MMA) was examined. Except blends containing P(VP-S) copolymers, blends of PSf with other copolymers were always immiscible. PSf and P(VP-S) copolymers formed miscible blends when copolymers have VP from 68 to $88 \mathrm{wt} \%$ and beyond these critical limits to form immiscible blends. Application of microporous PSf membrane for the ultrafiltration often limited because of its hydrophobic property. By Blending $\mathrm{P}(\mathrm{VP}-\mathrm{S})$ with PSf, we can provide hydrophilic property to the PSf membrane. Membranes prepared from PSf/P(VP-S) miscible blends showed performance advantage over PSf membrane.

\section{References}

(1) J. E. Harris and R. N. Johnson, Encyclopedia of Polymer Science and Engineering, ${ }^{\text {nd }}$ edn., H. F. Mark, N. M. Bikales, C. G. Overberger, and G. Menges, Eds., John Wiley, New York, 1988, Vol. 13, pp 196.
(2) S. Mok, D. J. Worsfold, A. Fouda, and T. Matsuura, J. Membrane Sci., 51, 193 (1994).

(3) C. M. Chan, Polymer Surface Modification and Characterization, Hanser Publishers, NY, 1993.

(4) M. Bryjak and I. Gancarz, Die Angew. Makromol. Chem., 219, 117 (1994).

(5) J. Hopkin and J. P. S. Badyal, Macromolecules, 27, 5498 (1994).

(6) K. S. Kim, K. H. Lee, K. Cho, and C. E. Park, J. Membr. Sci., 199, 135 (2002).

(7) M. Mulder, Basic Principle of Membrane Technology, Kluwer Academic Publishers, Dordrecht, 1996.

(8) T. A. Callaghan and D. R. Paul, J. Polym. Sci.: Part B, 32, 1847 (1994).

(9) K. E. Sickafus and A. M. Donald, Br. Polym. J., 21, 215 (1989).

(10) D. R. Paul and J. W. Barlow, Polymer, 25, 487 (1984).

(11) R. P. Kambour, T. J. Bendler, and R. C. Bopp, Macromolecules, 16, 1827 (1983).

(12) G. ten Brinke, F. E. Karasz, and W. J. Macknight, Macromolecules, 16, 1827 (1983).

(13) L. Zeman and D. Patterson, Macromolecules, 5, 513 (1972).

(14) M. J. Kim, J. E. Yoo, H. K. Choi, and C. K. Kim, Macromol. Res., 10, 91 (2002).

(15) S. Leob and S. Sourirajan, Adv. Chem. Ser, 38, 117 (1962).

(16) H. K. Lonsdale, J. Membr. Sci., 10, 81 (1982).

(17) H. Strathmann and K. Koch, Desalination, 21, 241 (1977). 